\title{
Distribution of $A B O$ and Rh-D Blood Group Antigens Among Blood Donors in the Amhara Regional State, Ethiopia
}

\author{
Bamlaku Enawgaw (D), Melak Aynalem (D), Mulugeta Melku (iD) \\ Department of Hematology and Immunohematology, School of Biomedical and Laboratory Sciences, College of Medicine and Health Science, \\ University of Gondar, Gondar, Ethiopia
}

Correspondence: Melak Aynalem, Department of Hematology and Immunohematology, School of Biomedical and Laboratory Sciences, College of Medicine and Health Science, University of Gondar, Gondar, 196, Ethiopia, Tel +25I-9126926I9, Email melak.aynalemI234@gmail.com

\begin{abstract}
Background: The ABO and Rh blood group systems are the most commonly used blood group systems in clinical settings. They are clinically significant for blood transfusion, organ transplantation, genetic studies, forensic determinations, and medico-legal issues. Therefore, this study aimed to determine the distribution of ABO and Rh-D blood groups among blood donors in the Amhara regional state, Ethiopia.
\end{abstract}

Methods: A cross-sectional study was conducted from March 2016 to September 2018 among blood donors in the four blood bank districts (Gondar, Bahir Dar, Debre Markos, and Dessie) of the Amhara regional state, Ethiopia. A total of 1040 blood donors aged from 18 years old to 60 years old were included using a simple random sampling technique. A structured questionnaire and a data collection sheet were used to obtain the socio-demographic data and blood group types of study participants. The ABO and Rh-D blood groups were typed using commercially prepared antisera after $1 \mathrm{~mL}$ of blood was taken from blood bags. The data was cleared by Epi-Info Version 7 and extracted into SPSS version 25 for analysis. The distribution of data was checked by using the Shapiro-Wilk test. Then, the data was presented in tables and figures.

Results: In this study, out of 1040 study participants, 55.6\% were males with a median age of 20 years (IQR $=19-24$ years). In this study, the distribution of ABO blood types O, A, B, and AB was 41.6\% (433/1040), 28.7\% (298/1040), 22.2\% (229/1040), and 7.7\% (80/1040), respectively. On the other hand, the Rh-D positivity was 92.5\% (962/1040).

Conclusion: The overall predominant $\mathrm{ABO}$ blood group in the Amhara regional state was $\mathrm{O}(41.6 \%)$, and the least was $\mathrm{AB}(7.7 \%)$. Besides, the overall Rh-D negative blood distribution was 7.5\%, which ranged from $4.46 \%$ to $9.6 \%$. Therefore, this information would be useful to the blood banks, especially in the planning of blood transfusion programmers.

Keywords: ABO, Rh-D, blood group, Amhara, Ethiopia

\section{Background}

Blood is the most essential body fluid, which is responsible for the transportation of nutrients, enzymes, and hormones throughout the body. ${ }^{1}$ Primarily, the blood has a fluid part called plasma and blood cells (white blood cells, red blood cells (RBC), and platelets). Of the cells, the RBC membrane is complex and contains numerous antigens that are made from glycoproteins and glycolipids. ${ }^{2}$ Based on the surface of RBC antigens, more than 100 blood group systems have been identified. However, the $\mathrm{ABO}$ and $\mathrm{Rh}$ blood group antigens are the most commonly used blood group antigens in a clinical setup and are used to avoid transfusion reactions and maternal death. ${ }^{3,4}$

The ABO blood groups were the first human blood group system that was discovered by Karl Landsteiner. The $\mathrm{ABO}$ antigens are controlled by 3 allelic genes placed on the long arm of chromosome $9 \mathrm{q}$ and are mainly responsible for the RBC membrane structural integrity and transportation of molecules through membranes. ${ }^{5,6}$ The ABO blood group is commonly tested for transfusion of blood and its components, organ transplantation, genetic studies, and forensic determinations, as well as for medico-legal issues like paternity testing. ${ }^{7}$ The $\mathrm{ABO}$ blood group can be 
categorized into four main blood groups. These are A, B, AB, and $\mathrm{O}$, which are encoded with N-acetyl D-galactose amine, D-galactose amine, both $\mathrm{N}$-acetyl D-galactose amine and D-galactose amine, and no antigen, respectively. However, the blood plasma contains reverse antibodies, which are targeted against particular antigens. ${ }^{4}$ The ABO blood group antibody occurs without any exposure to RBC by transfusion or pregnancy, called "natural" or "expected" antibodies. $^{7}$

In 1941, Landsteiner and Weiner discovered the Rhesus (Rh) blood grouping system. ${ }^{8}$ The Rh blood group is a multiantigen system expressed by 3 pairs of closely linked allelic genes located on chromosome 1, which consists of more than 50 antigen expressions. However, the major Rh antigens of medical importance are D, C, E, c, and e. ${ }^{5,9}$ An individual who has the Rh-D antigen is called Rh-D positive, while those lacking the antigen are called Rh-D negative. When a Rh-D negative person is transfused with Rh-D positive blood or a Rh-D negative mother is carrying a Rh-D positive fetus, the antigen will be recognized as a foreign particle by the immune system, which produces an antibody, resulting in a hemolytic transfusion reaction and hemolytic disease in the fetus and newborn. ${ }^{7}$

Knowledge of $\mathrm{ABO}$ and $\mathrm{Rh}$ blood group distribution will help to reduce maternal mortality rates through effective management of blood bank inventory. Blood bank access will also provide a safe and sufficient supply of blood, reducing the number of preventable disease deaths through effective management of blood bank inventory. Besides, the distribution of $\mathrm{ABO}$ and Rh-D blood groups varies in different populations (races, ethnic groups, and socio-economic classes). According to studies on $\mathrm{ABO}$ distribution in Ethiopia, the "O" blood group was the most dominant, followed by the "A" and "B" blood groups. ${ }^{110-15}$ The proportion of the "O" blood group ranged from $21.67 \%{ }^{16}$ to $47.04 \% .{ }^{15}$ On the other hand, the "AB" blood group was the least prevalent blood group type, which ranged from $2.87 \%{ }^{12}$ to $24.7 \% .{ }^{16}$ The Rh-D positive blood group was the most common, accounting for $92.77 \%{ }^{11}$ to $60.13 \%$ of the population. ${ }^{16}$ Even if studies on the $\mathrm{ABO}$ distribution have been conducted in Ethiopia, the distribution of $\mathrm{ABO}$ in the Amhara regional state at a regional level is not known. Therefore, this study aimed to determine the distribution of ABO and Rh-D blood group antigens among the Amhara regional state population.

\section{Materials and Methods}

\section{Study Setting and Study Population}

A population-based cross-sectional study was conducted from March 2016 to September 2018 to assess ABO and Rh-D blood group distribution in the Amhara regional state blood bank districts (Gondar, Bahir Dar, Debre Markos, and Dessie). The Amhara region has a land area estimated at 170,000 square kilometers. It is located in the northwestern part of Ethiopia between $8^{\circ} 45^{\prime}$ and $13^{\circ} 45^{\prime}$ North latitude and $36^{\circ} 20^{\prime}$ and $40^{\circ} 20^{\prime}$ East longitude. Based on the 2007 census conducted by the central statistical agency of Ethiopia, the Amhara regional state has a population of more than seventeen million people. Of those, 8,641,580 were men and 8,580,396 were women; urban inhabitants constituted around $12.27 \%$ of the population. ${ }^{17}$

A total of 1040 study participants aged from 18 years up to 60 years were included. All adult individuals living in the Amhara region were considered our source populations. While the blood donors who were coming to the blood bank center in the Amhara region for blood donation were considered as the study population, to select the study participants, a simple random sampling technique was used, and we used blood bank donor eligibility criteria. The blood banks select blood donors with nationally standardized criteria. According to these nationally harmonized criteria, blood donors' general good health shall be assessed through medical history, physical examination, and hemoglobin determination. Those with known diseases, those taking pharmacologically active substances or any prescription drugs, smokers, individuals who had major surgery in the past year, pregnant and lactating women, individuals who had donated blood in the previous 4 months and those who had received a blood transfusion in the previous year were excluded. Further, the blood donors should be between the ages of 18 and 65, weigh more than 45 kilograms, and have a normal hemoglobin value (13 for males and 12 for females), as well as normal vital signs (37 degrees Celsius, diastolic blood pressure of 70$90 \mathrm{mmHg}$, and systolic blood pressure of $100-150 \mathrm{mmHg}$ ). If the blood donor fulfilled the above-mentioned eligibility criteria, he/she could donate blood, and the donated blood was screened for infectious diseases like human immunodeficiency virus, Hepatitis B virus, Hepatitis C virus, and syphilis using ELISA techniques at each blood bank center. Study 
participants who volunteered to donate blood in Amhara Regional State were included in this study. However, study participants whose blood showed auto-control positivity were excluded from the study.

\section{Data Collection and Laboratory Procedures}

The structured questionnaires and a data collection sheet were used to obtain socio-demographic and laboratory data from study participants. The data was collected by trained nurses and laboratory technologists. First, training was given to the data collectors about the aim of the study. Then, the data collection procedure was collected under the supervision of a principal investigator.

\section{Socio-Demographic Data Collection}

A pre-tested structured questionnaire was used to collect sociodemographic data. To keep the quality of the data, the questionnaire was first written in the English language, then translated into the local language (Amharic), and finally back into the English language. To improve the quality of the data, the questionnaires were pre-tested in the Metema blood bank before the study began.

\section{Blood Sample Collection}

We follow the blood bank's procedure for blood collection and ABO and Rh-D blood grouping. First, the study participants were asked to give informed consent and undergo a physical examination for blood donation. Then the blood bank collected one unit of blood $(450 \mathrm{~mL})$ from eligible donors. A total of $1 \mathrm{~mL}$ of blood was collected from each blood unit for the $\mathrm{ABO}$ and $\mathrm{Rh}$ blood grouping tests. All the procedures were followed according to the Ethiopian National Blood Bank service protocols.

\section{$A B O$ and Rh-D Typing}

$\mathrm{ABO}$ and $\mathrm{Rh}-\mathrm{D}$ phenotyping were conducted using the slide method. One drop of whole blood was added to a clean slid and a commercially prepared standard antiserum (anti-A, anti-B, anti-D) with an equal amount was mixed. Then, after 2 minutes of incubation at room temperature, the results were recorded after looking with the naked eye and a microscope. The ABO and Rh-D blood group data were collected using a data collection format. To keep the quality of the result, known A-cell, B-cell, and Rh-D positive and negative cells were used.

\section{Data Entry and Analysis}

The data was entered into Epi-Info version 7. Then, it is transported to the SPSS version 25 software for the analysis. The study participants' age distribution was checked by the Shapiro-Wilk test. For normally distributed data, the mean and standard deviation were used, while for skewed data, the median and interquartile range (IQR) were used. Categorical variables were described using frequency and percentages.

\section{Ethics Approval}

Ethical clearance was obtained from the institutional review boards of the University of Gondar with a reference number of VP/RCS/05/19/2015. Written informed consent was taken from all study participants. Additionally, the confidentiality of information was assured by using codes for each specimen and the results. This study was conducted strictly following the Declaration of Helsinki.

\section{Results}

\section{Sociodemographic Characteristics of Study Participants}

In this study, a total of 1040 study participants were included. Of the total study participants, about 55.4\% (576/1040) were males, and the median age of the study participants was 20 years (IQR $=19-24$ years), with an age range of 18 to 61 years old. The majority of $79.5 \%(827 / 1040)$ and $87.1 \%$ (906/1040) of the study participants were students and single in their marital status, respectively (Table 1). 
Table I Sociodemographic Characteristics and Blood Group Distribution Among Blood Donors in Amhara Regional States

\begin{tabular}{|l|l|l|l|}
\hline Variables & Categories & Frequency & Percentages (\%) \\
\hline Sex & Male & 576 & 55.4 \\
& Female & 464 & 44.6 \\
& I8-20 years & 534 & 51.3 \\
& $21-25$ years & 323 & 31.1 \\
& $26-30$ years & 124 & 11.9 \\
Address & $>30$ years & 59 & 5.7 \\
& Gondar & 250 & 24.0 \\
& Debre Markos & 269 & 25.9 \\
Occupational status & Bahir Dar & 306 & 29.4 \\
& Dessie & 215 & 20.7 \\
& Student & 827 & 79.5 \\
& Employed & 144 & 13.8 \\
& Farmer & 2 & 0.2 \\
RH & Merchant & 58 & 5.6 \\
& Daily laborer & 6 & 0.6 \\
& Others & 3 & 0.3 \\
& Single & 906 & 87.1 \\
& Married & 97 & 9.3 \\
& A & 298 & 28.7 \\
& B & 229 & 22 \\
& AB & 80 & 7.7 \\
& O & 433 & 41.6 \\
& Rh positive & 962.5 \\
& Rh negative & 7.5 \\
\hline
\end{tabular}

\section{$A B O$ and Rh-D Blood Group Distribution of the Study Participants}

The distribution of ABO blood groups among Amhara regional state blood donors was 41.6\% (433/1040), 28.7\% (298/ 1040), 22\% (229/1040), and 7.7\% (80/1040) were O, A, B, and AB blood groups (Table 1).

Further, the combination of $\mathrm{ABO}$ and Rh blood group distribution was A Rh-D positive, A Rh-D negative, B Rh-D positive, B Rh-D negative, AB Rh-D positive, AB Rh-D negative, O Rh-D positive, and O Rh-D negative, with $26 \%$, $3 \%, 20 \%, 2 \%, 7 \%, 0 \%, 39 \%$, and 3\%, respectively. From districts, the predominant $\mathrm{ABO}$ blood group was the O blood type, and from the Rh blood group, the Rh-D positive was dominant in all districts (Figure 1).

In the Rh-D blood groups, the Rh-D positivity was $92.5 \%$ (962/1040) and the Rh-D negativity was 7.5\% (78/1040) (Figure 2). Further, the Rh-D negative blood group in Gondar, Debre-Markos, Bahir Dar, and Dessie was 9.6\% (24/250), $4.46 \%$ (12/269), 8.59\% (26/303), and 7.5\% (16/214) (Table 2).

\section{Discussion}

All humans have similar ABO and Rh blood group systems; while the difference is in the frequencies and distributions of blood types in different races, ethnic groups, and socio-economic groups or amongst different populations. Therefore, this study aimed to determine the distribution of $\mathrm{ABO}$ and $\mathrm{Rh}-\mathrm{D}$ blood group antigens among blood donors in Amhara regional state blood bank districts.

In the current study, the distribution of $\mathrm{O}, \mathrm{A}, \mathrm{B}$, and $\mathrm{AB}$ blood groups among Amhara regional state blood donors was $41.6 \%$ (433/1040), $28.7 \%$ (298/1040), 22.2\% (229/1040), and 7.7\% (80/1040), respectively. This showed that the "O" blood group was the dominant blood group, followed by A and B, and the least common blood group was AB. Also, the distribution of $\mathrm{ABO}$ in the blood bank districts is similar to the overall Amhara regional state distribution, where the "O" blood group is the dominant, followed by the "A" blood group, then the "B" blood group, and the "AB" blood group is the least common in all blood bank districts. This study is similar to a study conducted in India, ${ }^{18}$ Bangladesh, ${ }^{19}$ 


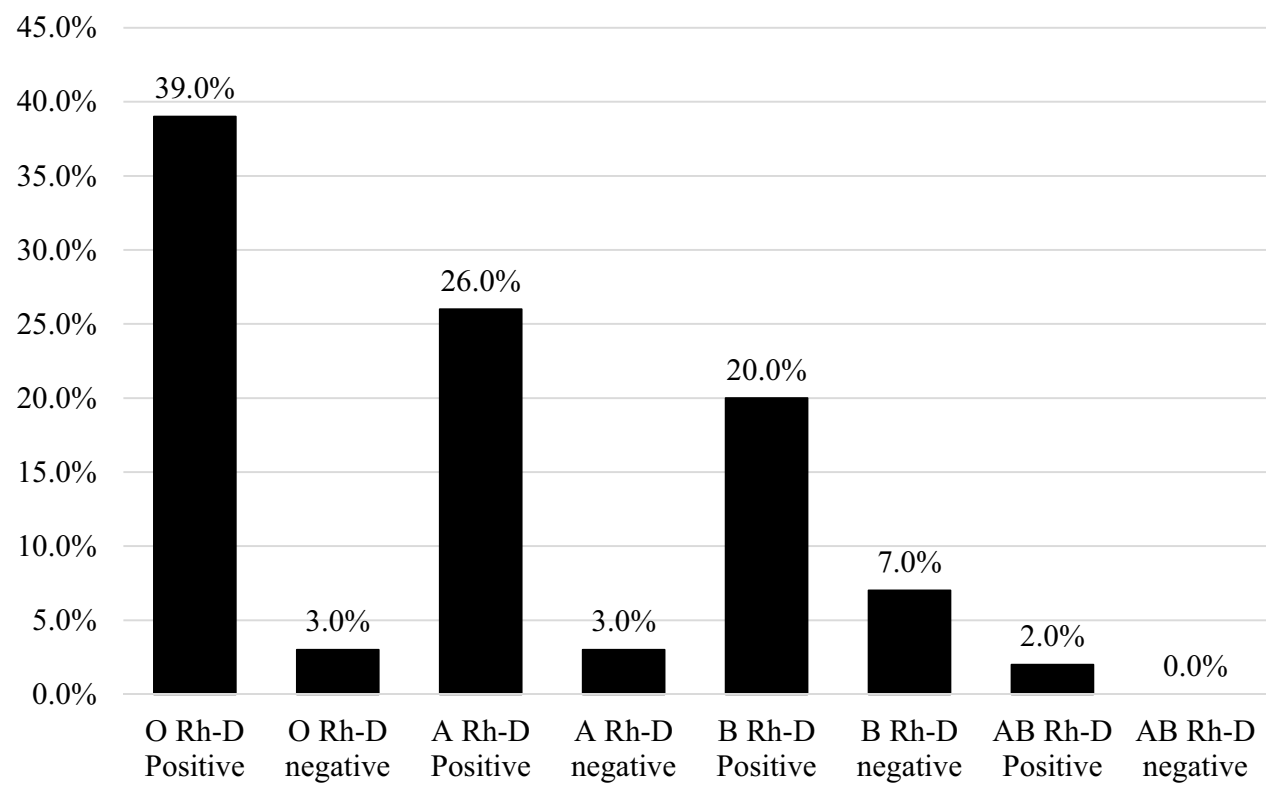

Figure I ABO and Rh-D blood group distribution among blood donors in Amhara regional states blood bank districts.

\section{Rh-D negative}

$7.5 \%$

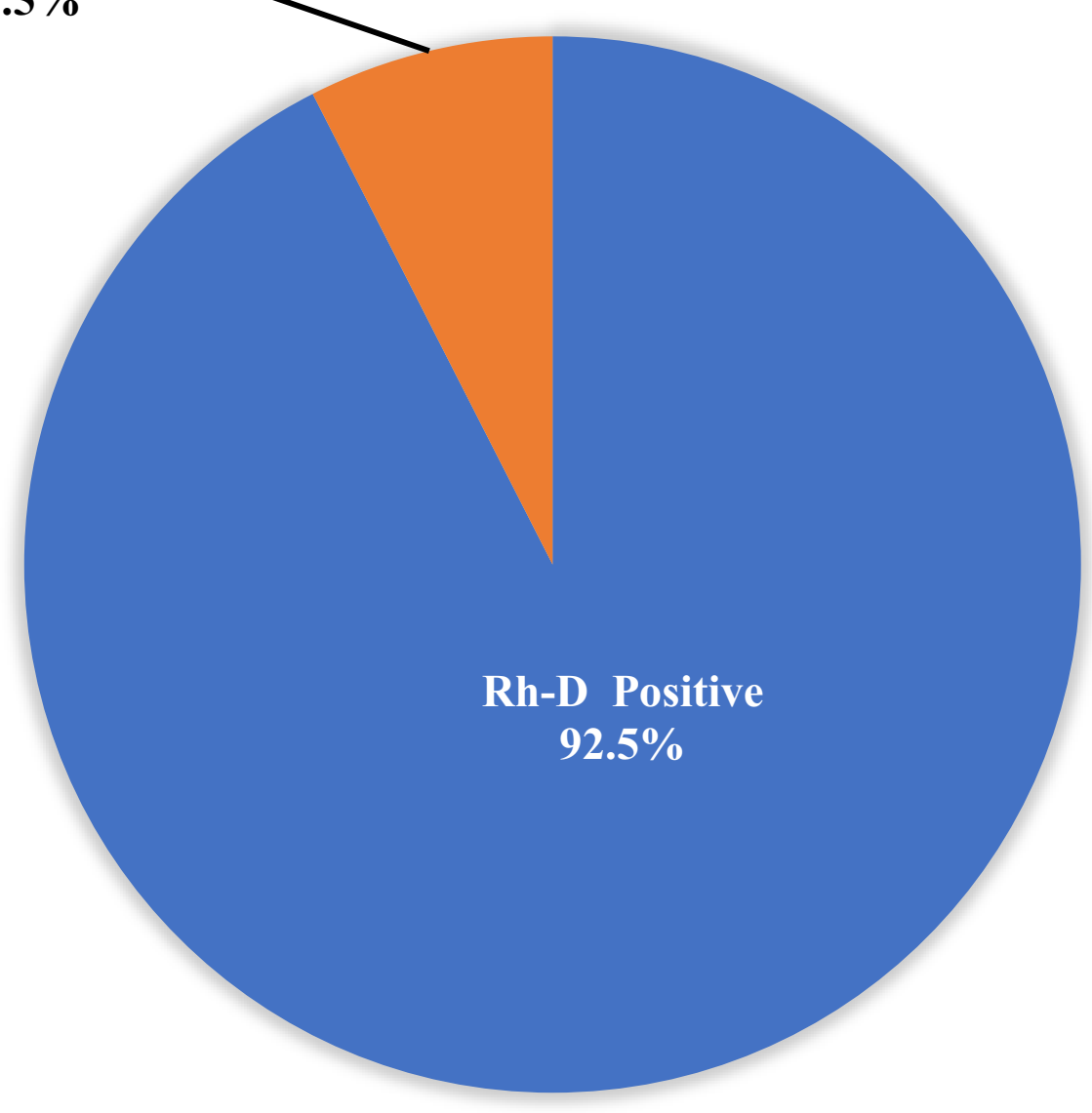

Figure 2 Rh-D blood group distribution among blood donors in Amhara regional states blood bank districts. 
Table 2 Sociodemographic Characteristics and Blood Group Distribution Among Blood Donors in Amhara Regional States Districts

\begin{tabular}{|c|c|c|c|c|c|}
\hline \multirow[t]{2}{*}{ Variables } & \multirow[t]{2}{*}{ Categories } & \multicolumn{4}{|c|}{ Blood Donors Categorized by District } \\
\hline & & Gondar $(n=250)$ & Bahir Dar $(n=306)$ & Debra-Markos (n = 269) & Dessie $(n=215)$ \\
\hline \multirow[t]{2}{*}{ Sex } & Male & $124(49.6 \%)$ & 175 (57.2\%) & $157(58.4 \%)$ & $120(55.8 \%)$ \\
\hline & Female & $126(50.4 \%)$ & $|3|(42.8 \%)$ & $112(36.6 \%)$ & 95 (944.2\%) \\
\hline \multirow[t]{2}{*}{ Marital Status } & Single & $240(26 \%)$ & $286(31 \%)$ & $25 ।(27.2 \%)$ & $146(15.8 \%)$ \\
\hline & Married & $10(8.5 \%)$ & $20(17.1 \%)$ & 18 (I5.4\%) & $69(59 \%)$ \\
\hline \multirow[t]{4}{*}{ Age } & $18-20$ years & $129(24.2 \%)$ & $202(37.8 \%)$ & $146(27.3 \%)$ & $57(10.7 \%)$ \\
\hline & $21-25$ years & 99 (30.7\%) & $77(23.8 \%)$ & $76(23.5 \%)$ & 71 (22\%) \\
\hline & $26-30$ years & $19(15.3 \%)$ & $24(19.4 \%)$ & $33(26.6 \%)$ & $48(38.7 \%)$ \\
\hline & $>30$ years & $3(5.1 \%)$ & $3(5.1 \%)$ & 14 (23.7\%) & 39 (66.1\%) \\
\hline \multirow[t]{6}{*}{ Occupation } & Student & $230(27.8 \%)$ & 269 (32.5\%) & $240(29 \%)$ & $88(10.6 \%)$ \\
\hline & Employed & $9(6.3 \%)$ & $34(23.6 \%)$ & 29 (20.1\%) & $72(50 \%)$ \\
\hline & Farmer & 0 & I (50\%) & 0 & I (50\%) \\
\hline & Merchant & $6(10.3 \%)$ & 0 & 0 & $52(89.7 \%)$ \\
\hline & Daily laborer & $4(66.7 \%)$ & 0 & 0 & $2(33.3 \%)$ \\
\hline & Others & I (33.3\%) & $2(66.7 \%)$ & 0 & 0 \\
\hline \multirow[t]{4}{*}{ ABO Blood group } & $A$ & $80(26.8 \%)$ & 77 (25.8\%) & 79 (26.5\%) & $62(20.8 \%)$ \\
\hline & B & $60(26.2 \%)$ & 70 (30.6\%) & 49 (2I.4\%) & $50(21.8 \%)$ \\
\hline & $A B$ & I8 (22.5\%) & 25 (31.3\%) & 25 (31.3\%) & $12(15 \%)$ \\
\hline & O & $92(21.2 \%)$ & 134 (30.9\%) & $116(26.8 \%)$ & $91(21 \%)$ \\
\hline \multirow[t]{2}{*}{ Rh-D Blood group } & Positive & $226(23.5 \%)$ & $280(29.1 \%)$ & $257(26.7 \%)$ & 199 (20.7\%) \\
\hline & Negative & $24(30.8 \%)$ & $26(33.3 \%)$ & 12 (I5.4\%) & $16(20.5 \%)$ \\
\hline
\end{tabular}

Nigeria, ${ }^{20-22}$ Uganda, ${ }^{6}$ and Ethiopia (Jimma, ${ }^{13}$ Gambella, ${ }^{14}$ and Bahir-Dar). ${ }^{10}$ The studies indicated that the "O" blood group was the most common, and $\mathrm{AB}$ was the least common. In contrast to this, a study conducted in India ${ }^{23}$ and Pakistan $^{24,25}$ showed that the "B" blood group was dominant, followed by the "O" blood group. Similarly, a study conducted in Woldia, Ethiopia ${ }^{16}$ showed that the A blood group was the predominant blood group compared to others. The discrepancy between our findings and those of other studies could be attributable to differences in sample size, sampling technique, and genetic variation across ethnic groups within the Amhara region's population and populations in other nations.

In the current study, the Rh-D positive blood group was $92.5 \%$ (95\% CI: 92.49, 92.51) and the Rh-D negative blood group was $7.5 \%$ (95\% CI: 7.495, 7.505). This showed that nearly ten times more of the Rh-D positive blood group type was collected from blood donors than the Rh-D negative blood group types. This indicated that Rh-D negative was the least common blood group type. This study was nearly similar to the study conducted in Jimma ${ }^{13}$ in which 7.2\% of Rh-D negative blood types were identified. In contrast, this study's finding was higher than a study conducted in Bangladesh, ${ }^{19}$ India, ${ }^{23}$ Nigeria, ${ }^{21,22,26}$ Uganda, ${ }^{6}$ and Niger ${ }^{21}$ which ranged from $1.2 \%$ to $6.55 \%$. In contrast, this study is lower than a study conducted in India, ${ }^{18}$ and different parts of Ethiopia (Woldia, ${ }^{16}$ Gambella, ${ }^{14}$ and Bahirdar ${ }^{10}$ ) showed that $8.9 \%$, $39.86 \%, 19.37 \%$, and $8.5 \%$ were Rh-D negative, respectively. Further, in this study, the Rh-D negative blood group in Gondar, Debre-Markos, Bahir Dar, and Dessie districts was 9.6\% (24/250), 4.46\% (12/269), 8.59\% (26/303), and 7.5\% (16/214), respectively. This showed that the Rh-D negative blood group ranges from $4.46 \%$ to $9.6 \%$.

In this study, the combination of $\mathrm{ABO}$ with the Rh blood group distribution among the study participants was as follows: A Rh-D positive, A Rh-D negative, B Rh-D positive, B Rh-D negative, AB Rh-D positive, AB Rh-D negative, O Rh-D positive, and O Rh-D negative were $26 \%, 3 \%, 20 \%, 2 \%, 7 \%, 0 \%, 39 \%$, and $3 \%$, respectively. This showed that the "O" positive and " $\mathrm{A}$ " positive blood groups were the first leading blood types, and the "AB" negative and "O" negative blood groups were the least common blood group. However, the $\mathrm{O}$ Rh-D negative blood group was the universal cell donor that can help many patients during emergency transfusion, but of the total donors, only 3\% (31/1040) were "O" Rh-D negatives, so the blood bank should be aware of this and the blood should be used wisely. This study is similar to a study conducted in Bahir-Dar. ${ }^{10}$ "O" Rh-D positive and "A" were the first two dominant blood groups, while "AB" 
Rh-D negative and "O" Rh-D negative were the least dominant blood types among blood donors. In contrast to this, a study conducted in India ${ }^{23}$ and Pakistan ${ }^{24,25}$ showed that "B" positive was the dominant blood group followed by the "O" positive blood group. The variation of this study with other studies might be related to the difference in sample size, diagnostic methods, and genetics among the ethnic groups studied populations.

\section{Conclusion and Recommendations}

In this study, the predominant blood group type was "O" and the least was $\mathrm{AB}$ among blood donors in the Amhara regional state blood bank districts. Besides, the Rh-D negative blood distribution in the Amhara regional state blood bank districts was $7.5 \%$, and its range was from $4.46 \%$ to $9.6 \%$ in all the blood districts included in this study. Therefore, this information would be beneficial to blood banks, particularly in the development of blood transfusion programs for the most efficient use of blood collected from donors.

\section{Abbreviations}

EDTA, ethylene di-amine tetra acetic acid; IQR, interquartile range; RBC, red blood cell; Rh, rhesus; UoG-CSH, University of Gondar Comprehensive Specialized Hospital.

\section{Data Sharing Statement}

All data generated and/or analyzed in this study are available within the manuscript.

\section{Acknowledgments}

We would like to thank the study participants who participated in this study, and we would like to acknowledge the regional health office for providing the necessary information during the study.

\section{Author Contributions}

All authors made substantial contributions to conception and design, acquisition of data, or analysis and interpretation of data; took part in drafting the article or revising it critically for important intellectual content; agreed to submit it to the current journal; gave final approval of the version to be published, and agreed to be accountable for all aspects of the work.

\section{Disclosure}

The authors declare that there is no conflicts of interest regarding the publication of this manuscript.

\section{References}

1. Tesfaye K, Petros Y, Andargie M. Frequency distribution of ABO and Rh (D) blood group alleles in Silte Zone, Ethiopia. Egypt J Med Hum Genet. 2015;16:71-76. doi:10.1016/j.ejmhg.2014.09.002

2. Dzieczkowski JS, Anderson KC. Blood Group Antigens and Therapy in Harrison's Principles of International Medicine. 14th ed. New York: McGraw Hill; 1998.

3. Egesie UG, Egesie OJ, Usar I, Johnbull TO. Distribution of ABO, rhesus blood groups and hemoglobin electrophoresis among the undergraduate students of Niger delta university Nigeria. Niger J Physiol Sci. 2008;23(1):5-8. doi:10.4314/njps.v23i1-2.54900

4. Singh S, Arora I. Frequency and distribution of ABO and rhesus (D) blood groups in district Chamba, Himachal Pradesh: a study from rural tertiary care hospital. Int J Community Med Public Health. 2018;5(2):689-692. doi:10.18203/2394-6040.ijcmph20180251

5. Jahanpour O, Jeremia J, Ernest O, Mremi A, Shao E. ABO and Rhesus blood group distribution and frequency among blood donors at Kilimanjaro Christian Medical Center, Moshi, Tanzania. BMC Res Notes. 2017;10(738). doi:10.1186/s13104-017-3037-3

6. Apecu RO, Mulogo EM, Bagenda F, Byamungu A. ABO and Rhesus (D) blood group distribution among blood donors in rural south western Uganda: a retrospective study. BMC Res Notes. 2016;9:513. doi:10.1186/s13104-016-2299-5

7. Suresh B, Babu S, Chandra P, et al. Distribution of ABO and rhesus (D) blood group antigens among blood donors at a tertiary care teaching hospital blood bank in south India. J Clin Sci Res. 2015;4:129-135. doi:10.15380/2277-5706.JCSR.14.064

8. Firkin F, Chesterman C, Penington D, Rush B. de Gruchy's clinical Hematology in medical practice. In: Blood Groups; Blood Transfusion; Acquired Immune Deficiency Syndrome. 5th ed. NewDelhi: Oxford University Press; 1989:475-496.

9. Behra D, Joshi D. Distribution of ABO blood group and RH (D) factor in Western Rajasthan. J Med Res. 2013;3:73-75.

10. Legese B, Shiferaw M, Tamir W, Tiruneh T. Distribution of ABO and Rhesus blood group phenotypes among blood donors at Bahir Dar Blood bank, Amhara, Northwest Ethiopia: a retrospective cross-sectional study. J Blood Med. 2021;12:849. doi:10.2147/JBM.S329360 
11. Tiruneh A, Yetneberk T, Gelaw M, Eshetie D. Frequency of ABO and Rh blood group distribution at Debre Tabor blood bank, Amhara Region, North-Central Ethiopia. A six-year retrospective survey. J Blood Med. 2020;11:357.

12. Atire FA. Distribution of $\mathrm{ABO}$ and Rh blood groups among students of some ethnic groups at Dilla University, Ethiopia. Int J Genet Genomics. 2015;3(1):8. doi:10.11648/j.ijgg.20150301.12

13. Zerihun T, Bekele S. Pattern of ABO and Rhesus blood groups distribution of five years survey in Jimma Town blood bank, South West Ethiopia. J Health Educ Res Dev. 2016;4:1-4.

14. Golassa L, Tsegaye A, Erko B, Mamo H. High rhesus (Rh (D)) negative frequency and ethnic-group based ABO blood group distribution in Ethiopia. BMC Res Notes. 2017;10(1):1-5. doi:10.1186/s13104-017-2644-3

15. Woldu B, Melku M, Shiferaw E, Biadgo B, Abebe M, Gelaw Y. Phenotype, Allele and genotype frequency of ABO and Rhesus D blood groups of blood donors at the North Gondar district blood bank, Northwest Ethiopia. J Blood Med. 2022;13:11. doi:10.2147/JBM.S346904

16. Abegaz SB. ABO and Rh (D) blood group distribution among blood donors: a study of natural and computational science faculty graduating class students at Woldia University, Ethiopia. Int J Genet Mol Biol. 2021;13(1):21-30. doi:10.5897/IJGMB2020.0203

17. Population Census Commission. Summary and statistical report of the 2007 population and housing census; 2008:314.

18. Sharma DC, Rai S, Iyenger S, Jain B, Sao S. Prevalence and distribution of ABO and Rh-D antigens along with its subgroups \& rare types in Greater Gwalior region; 2013.

19. Talukder S, Das R. Distribution of ABO and Rh blood groups among blood donors of Dinajpur district of Bangladesh. Dinajpur Med Col J. 2010;3 (2):55-58.

20. Odokuma E, Okolo A, Aloamaka P. Distribution of ABO and rhesus blood groups in Abraka, Delta state. Niger J Physiol Sci. 2007;22(1-2):89-91.

21. Enosolease ME, Bazuaye GN. Distribution of ABO and Rh-D blood groups in the Benin area of Niger-Delta: implication for regional blood transfusion. Asian J Transfus Sci. 2008;2(1):3. doi:10.4103/0973-6247.39502

22. Erhabor O, Isaac I, Saidu A, et al. The distribution of ABO and Rhesus blood groups among residents of Gusau, Zamfara State, North Western Nigeria. Res Rev J Med Health Sci. 2013;2(4):58-63.

23. Barot T, Patel D, Shah R. Distribution of $\mathrm{ABO}$ and Rhesus (Rh) blood groups among voluntary blood donors in Central Gujarat, India. Int $j$ Contemp Med Res. 2020;7(7):61-63.

24. Khattak ID, Khan TM, Khan P, Shah SMA, Khattak ST, Ali A. Frequency of ABO and Rhesus blood groups in District Swat, Pakistan. J Ayub Med Coll Abbottabad. 2008;20(4):127-129.

25. Pramanik T, Pramanik S. Distribution of ABO and Rh blood groups in Nepalese medical students: a report. East Mediterr Health J. 2000;6 (1):156-158. doi:10.26719/2000.6.1.156

26. Bamidele O, Arokoyo DS, Akinbola AO. Distribution of ABO and rhesus blood groups among medical students in Bowen University, Iwo, Nigeria. Ann Biol Res. 2013;4(11):1-6.

\section{Publish your work in this journal}

The Journal of Blood Medicine is an international, peer-reviewed, open access, online journal publishing laboratory, experimental and clinical aspects of all aspect pertaining to blood based medicine including but not limited to: Transfusion Medicine; Blood collection, Donor issues, Transmittable diseases, and Blood banking logistics; Immunohematology; Artificial and alternative blood based therapeutics; Hematology; Biotechnology/nanotechnology of blood related medicine; Legal aspects of blood medicine; Historical perspectives. The manuscript management system is completely online and includes a very quick and fair peer-review system. Visit http://www.dovepress.com/testimonials.php to read real quotes from published authors.

Submit your manuscript here: http://www.dovepress.com/journal-of-blood-medicine-journal 